\section{Rules on originality need to be clearly set out}

Sir - Japan's Institute of Physical and Chemical Research, RIKEN, has now published its investigation into US accusations of espionage against Takashi Okamoto, head of the institute's laboratory for neurodegeneration signalling (see Nature 411, 225; 2001 and Nature 411, 991; 2001).

RIKEN's report, whose main author is chief executive Shun-ichi Kobayashi, concludes that there is no evidence that the institute intentionally planned to bring in the materials from the United States. But Okamoto has remained silent on the issue, and has now abruptly left RIKEN.

This affair has shocked Japanese scientists and highlights the differences in definitions of 'originality' between Japan and the United States. The United States encourages excellent scientists from all over the world to work there on short-term contracts, and is understandably keen to maintain its competitive edge. But to avoid similar misunderstandings in future, the US government should issue clear guidelines for foreign researchers about originality and authorization for removal of research materials.

Tadasu Nagaoka, Hisatsugu Miyakoshi Kouseiren Takaoka Hospital, Eiraku 5-10, Takaoka, Toyama, 933-8555, Japan

\section{Related problems}

Sir - In his Words essay "Yes, but what's it for?” (Nature 412, 771; 2001), Steve Blinkhorn reminds us that the current state of language can make it difficult to discuss evolution in an accurate way. We must be constantly alert to invocation of teleological explanations when describing evolution.

I believe that another (related) problem lies in the inherent ambiguity of the term 'related', used to depict similarity between biological objects such as nucleic acid or protein sequences. It is common to see very similar sequences described as "closely related", less similar ones as "distantly related" and dissimilar ones as "unrelated". Of course, the truth is that related (similar) sequences are not always related in terms of shared evolutionary origin (homology), and vice versa.

This ambiguity in meaning of the word 'related' differs from that which some believe exists for 'homology'. It is generally agreed that the only useful definition of homology between biological objects is "having a common evolutionary origin" (G. R. Reeck et al. Cell 50, 667; 1987). But when using the term 'related' in a biological context, it would be helpful to readers if authors could be explicit about which meaning is appropriate.

Alex C. W. May

Division of Mathematical Biology, National Institute for Medical Research, The Ridgeway, Mill Hill, London NW7 1AA, UK

\section{Give us time to put reforms into action}

Sir — In your News feature "Forza Scienza!” (Nature 412, 264-265; 2001), you discuss the scientific management of Italy's Higher Institute of Health (ISS) in
Rome since its reform. Yet this reform will not be operative until the end of the year, when the rules and regulations set down in March will be approved. Therefore it would seem wiser and more appropriate to leave us some time to implement the reform before quoting statements such as "The situation [at ISS] is worse than ever".

The whole staff, with me at the front line, is strongly committed to exploit all the autonomy and flexibility provided by the new rules to allow us to strengthen our research and output in terms of public health. This is our only mission.

Enrico Garaci

ISS, Viale Regina Elena 299, 00161 Rome, Italy

\title{
In the stem-cell debate, new concepts need new words
}

\section{Confusion arises when the existing vocabulary we use is inadequate to describe new methodologies.}

Sir - Your Opinion article "The meaning of life" (Nature 412, 255; 2001) implied that the suggestion made by Advanced Cell Technology's ethics advisory board, to substitute the term 'ovumsum' for 'embryo' in the case of eggs activated following nuclear transfer, is an attempt to sidestep the moral issue associated with deriving stem cells from human embryos. This implication is not correct.

I am the board member who derived the term 'ovasome' (not 'ovumsum') for reasons of accuracy of language, not out of a desire to sidestep controversial issues. Much of the current confusion in the US Congress stems from describing new methodologies with existing language.

The heart of the matter rests with the wondrous properties of eggs. Because, as you point out in your article, neither eggs nor sperm are dead before fertilization, a compelling argument has been made that fertilization is not the beginning of life, but a continuum of life in a new form. The real issue is, therefore, the perceived threat to the sanctity of the union of egg and sperm to create a new individual.

Unfortunately, the union of human eggs and sperm fails far more often than it succeeds in producing a new being. It is this high failure rate, and the inability to prevent it, that has led to the horrifying stores of frozen human embryos worldwide. Rather than asking if these frozen embryos can be used to derive stem cells, we should be asking why so many were created. And should more be created for the sole purpose of deriving stem cells, as was done recently in a Virginia clinic?

The answer is no. Other technologies can be developed to produce stem cells with greater medical advantage. Stem-cell therapy holds the promise of replacing defective cells in adult organs, and the optimal way to attempt this medically is with cells from the affected individual. Eggs are capable, by an as-yet unknown mechanism, of remodelling the nucleus of an adult cell into the much larger, more open format of a 'pronucleus', the first nucleus formed by an activated egg. This yields a new cell with vastly expanded potential to develop into a variety of cell types: this is the power to be harnessed.

An alternative approach is to activate the egg with its own genetic material intact (parthenogenesis). Stem cells derived from parthenotes will have half as many problems of tissue rejection as stem cells derived from eggs fertilized by sperm, which will have the same tissue compatibility problems as transplanted organs.

The early stages of developing these procedures may be similar to those used to clone individuals, but as time goes on the efficiency of creating genetically matched stem cells from activated eggs will increase, and the potential for development to an offspring will decrease. It follows that if creating an offspring is not the goal, the term 'embryo' is not accurate. 'Cloning' is more accurate, but is broadly used to describe a variety of experimental procedures. Hence my suggestion of 'ovasomagenesis' to describe the process and 'ovasome' to describe the product of activating an egg to become a somatic cell. Ann A. Kiessling Harvard Medical School, Blackfan Circle, Boston, Massachusetts 02115, USA 